\title{
Research on Identification Technology of Explosive Vibration Based on EEMD Energy Entropy and Multiclassification SVM
}

\author{
Huayuan Ma, Xinghua Li $\mathbb{D}^{D}$, Qiang Liu $\mathbb{D}^{D}$, Xie Xingbo, Chong Ji, and Changxiao Zhao
}

Army Engineering University of PLA, Nanjing, China

Correspondence should be addressed to Xinghua Li; 379523589@qq.com and Qiang Liu; 3453815448@qq.com

Received 5 January 2020; Revised 16 March 2020; Accepted 20 July 2020; Published 8 August 2020

Academic Editor: Jiawei Xiang

Copyright $\odot 2020$ Huayuan Ma et al. This is an open access article distributed under the Creative Commons Attribution License, which permits unrestricted use, distribution, and reproduction in any medium, provided the original work is properly cited.

\begin{abstract}
In this study, the authors introduced energy entropy as a reference feature into the field of blast vibration recognition classification and achieved good results. On the basis of the previous experimental database, 4 kinds of typical vibration signals were selected to form the sample group (building collapse vibration, surface rock blast vibration, underground tunnel blast vibration, and natural gas pipeline explosion vibration). EEMD (ensemble empirical mode decomposition) algorithm was used to calculate the energy entropy of each signal. Taking eigenvector composed of CEE (components of energy entropy) as input, multiclassification SVM algorithm was used for training and prediction. Prediction accuracy was more than $80 \%$. Compared with BP (backpropagation) neural network algorithm, SVM (support vector machine) algorithm has higher training efficiency. The research results can be used in urban vibration monitoring, identify the nature of vibration source in time, and provide technical support for rapid response of emergency rescue.
\end{abstract}

\section{Introduction}

The blasting vibration signal is a typical short-time nonstationary random signal. The characteristics of the blasting vibration signal can reflect the blasting type, blasting parameters, and site media information. The feature extraction of blasting vibration signal is the basis of studying blasting vibration prediction, structural response under blasting vibration excitation, and reducing blasting vibration. At present, the characteristics of blasting vibration signals mainly include peak characteristics, spectral features, timeholding characteristics, and energy characteristics. A large number of scholars have carried out in-depth research on the influence of characteristics and blasting parameters on signal characteristics. Min et al. [1] studied the strain law of buried pipeline under collapse vibration load. Through range analysis and variance significance analysis, it was found that the dynamic strain of buried pipeline under impact was more significant than that of collapse height and self-weight. Lu [2] obtained the energy distribution of each frequency band after multiscale lifting wavelet packet decomposition of the measured blasting vibration signal and summarized the energy distribution characteristics of the frequency band of the blasting vibration signal. Based on the dimension analysis theory, Zhong [3] discussed the influence factors of blasting vibration holding time and deduced the prediction formula of blasting vibration holding time.

With the deepening of research, a new branch of blasting vibration characteristics has appeared: energy entropy. Shannon referred to the concept of thermodynamics and called the average amount of information excluding redundancy "energy entropy" [4]. Energy entropy is an important concept in information theory and a description of the degree of system uncertainty. It can reflect the degree of random change of signals. In recent years, it has been widely used in mechanical fault diagnosis [5-7], speech signal processing [8], mine micro-earthquake signal recognition $[9,10]$, and other fields [11-13]. In this paper, EEMD algorithm was used to decompose the energy entropy of vibration signal, and the energy characteristics of each IMF are obtained as the input vector of SVM. Through the analysis and training of many kinds of vibration signals, the accurate prediction of vibration types was realized. The research results can provide technical reserves for urban vibration 
monitoring system and quick and accurate information of explosion source types for decision-makers of disaster prevention and mitigation command departments.

\section{EEMD Energy Entropy}

The EEMD (ensemble empirical mode decomposition) algorithm is a mature nonstationary random signal decomposition algorithm based on the EMD (empirical mode decomposition) algorithm. The original intention is to decompose any nonstationary random signal into multiple IMFs (intrinsic mode function), so as to provide feasibility for further Hilbert transform. EMD algorithm is different from the traditional wavelet and Fourier transform, its decomposition process has no fixed base, it has adaptive characteristics, and so it is famous for its efficiency and accuracy. But, the frequency aliasing effect of EMD algorithm has been perplexing scholars. The improved idea of EEMD is to add white noise at the beginning of EMD decomposition cycle, so as to solve the problem of frequency aliasing in traditional EMD algorithm.

The signal was decomposed by the EEMD algorithm to obtain a plurality of IMFs, which represent the characteristic distribution of the signal energy. Due to the difference of explosion source, initiation mode, and propagation medium of different types of explosion vibration signals, the energy distribution characteristics of IMFs are different.

Here we introduce the concept of EEMD energy entropy:

$$
H_{E n}=-\sum_{i=1}^{n} \frac{E_{i}}{E} \log \frac{E_{i}}{E},
$$

where $E$ is the total energy of the signal and $E_{\mathrm{i}}$ is the energy of each IMF. In this paper, $-E_{i} / E \log E_{i} / E$ is called CEE (components of energy entropy).

Several vibration signals from different sample groups are selected for decomposition, and their respective energy entropies are calculated, as shown in Table 1. The signal acquisition parameters are shown in Table 2. A simple statistical analysis of the energy entropy data of four kinds of vibration shows that their statistical characteristics are significantly different. Therefore, the vibration energy entropy can be studied in depth as a characteristic parameter of vibration signal classification.

\section{Multiclassification SVM}

Support vector machine is a binary linear classifier with supervised learning mode [14]. As shown in the Figure 1, for a given input, the sample set $S$ is linearly separable. Each sample contains multiple features, thus forming a feature space (2dimensional space shown in the figure). The goal of the algorithm is to find the hyper plane with the largest margin $\omega \mathrm{X}+b=0$ [15]. The decision boundary satisfying the condition actually constructs two parallel hyperplanes as the interval boundary to distinguish the classification of samples [16]:

$$
\begin{array}{r}
\omega X+b \geq 1, \Rightarrow y=1, \\
\omega X+b \leq 1, \Longrightarrow y=-1 .
\end{array}
$$

All samples above the upper interval boundary belong to a positive class and those below the lower interval boundary belong to a negative class. The distance $2 / \omega$ between two interval boundaries is defined as the margin [17]. The samples of positive and negative classes on the boundary of interval are support vectors.

Support vector machine uses the hinge loss function to calculate the empirical risk. It adds the regularization module to optimize the structural risk and has good robustness [18]. For the nonlinear classification problem, SVM can learn through kernel function method, and the classification effect is still excellent [19].

As mentioned above, SVM is initially a linear classifier. With the in-depth study of scholars, SVM algorithm has been successfully extended to the field of nonlinear separability [20]. The principle is to map the nonlinear separable problem from the original feature space to a higher dimensional Hilbert space by using nonlinear functions [21]. There are hyperplanes [22] in space that can separate positive classes from negative classes as shown in Figure 2.

The standard SVM computing process was used to construct multiple decision boundaries in order to achieve multiclassification of samples [23]. The commonly used structures are "one-to-all (OAA-SVM)" and "one-to-one (OAO-SVM)". OAA-SVM establishes $M$ decision boundaries for $M$ classifications, and each decision boundary determines the attribution of one classification to all other classifications. OAO-SVM used in this paper is a voting method [24]. The calculation process is to establish decision boundaries for any two of the $M$ classifications, that is, there are $M(M-1) / 2$ decision boundaries in total. The final sample category is determined by the score order of decision boundary.

\section{Application of Experimental Data Analysis}

In this paper, vibration data under four typical explosion scenarios are selected.

Scene 1: pipeline explosion vibration: at present, the global natural gas pipeline construction mileage has exceeded $370000 \mathrm{~km}$ [25]. The pressure of pipeline operation keeps increasing. Due to various reasons, natural gas pipeline explosion accidents occur frequently, which brings hidden dangers to the production safety of human society. Therefore, the author has carried out many full-scale highpressure natural gas pipeline explosion tests, as shown in Figure 3, and recorded the pipeline explosion vibration data as a kind of sample for this study.

Scene 2: building collapse vibration: with the expansion of the city scale and the exhaustion of the design life of the building itself, the blasting demolition engineering of the high-rise building in the city is increasing day by day. The high-rise building disintegrates in the process of collapse, which causes violent vibration when hitting the ground, a serious threat to surrounding buildings. The research team collected a number of groups of building collapse vibration data as a kind of sample for this study, as shown in Figure 4.

Scene 3: surface rock blast vibration: rock blasting is a key method for the rapid and high-quality construction of 
TABLE 1: Comparison of energy entropy of typical vibration signals.

\begin{tabular}{|c|c|c|c|c|c|}
\hline \multicolumn{2}{|c|}{ Signal type } & \multirow{2}{*}{$\begin{array}{c}\begin{array}{c}\text { Pipeline explosion } \\
\text { vibration }\end{array} \\
2.1656\end{array}$} & \multirow{2}{*}{$\begin{array}{c}\text { Underground tunnel } \\
\text { blast vibration }\end{array}$} & \multirow{2}{*}{$\begin{array}{c}\begin{array}{c}\text { Demolition collapse } \\
\text { vibration }\end{array} \\
1.5578\end{array}$} & \multirow{2}{*}{$\begin{array}{c}\text { Surface rock blast vibration } \\
1.5580\end{array}$} \\
\hline \multirow{4}{*}{ Energy entropy } & Signal 1 & & & & \\
\hline & Signal 2 & 2.2454 & 2.3216 & 1.0854 & 1.9108 \\
\hline & Signal 3 & 2.0622 & 2.2722 & 1.6836 & 1.7539 \\
\hline & Signal 4 & 2.4368 & 2.3813 & 1.6146 & 2.0222 \\
\hline \multicolumn{2}{|c|}{ Expectation } & 2.2275 & 2.2260 & 1.4854 & 1.8112 \\
\hline \multicolumn{2}{|c|}{ Variance } & 0.1372 & 0.1758 & 0.2352 & 0.1745 \\
\hline
\end{tabular}

TABle 2: Parameters of recorder.

\begin{tabular}{lcccc}
\hline Type of recorder & Type of sensor & Number of channels & Frequency response range/Hz & Sampling rate/SPS \\
\hline TC-4850 & Piezoelectric & 3 & $5 \sim 500$ & 8000 \\
Blast-UM & Piezoelectric & 3 & $5 \sim 300$ & 10000 \\
\hline
\end{tabular}

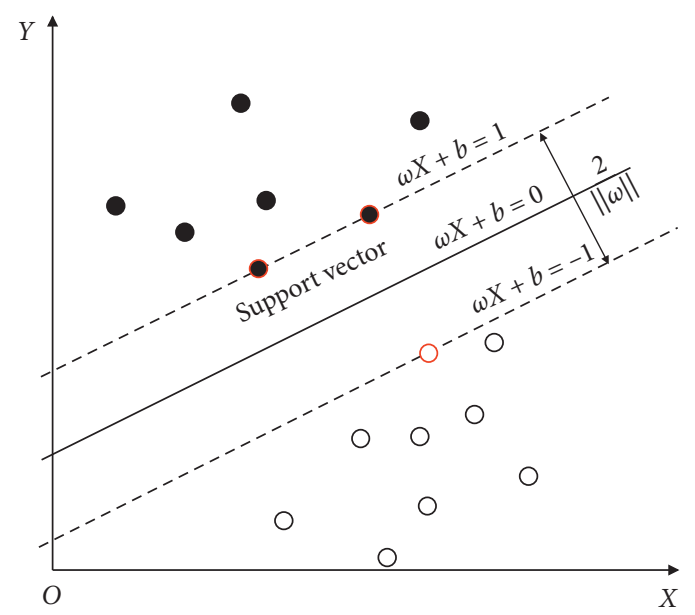

FIgURE 1: Schematic diagram of SVM.

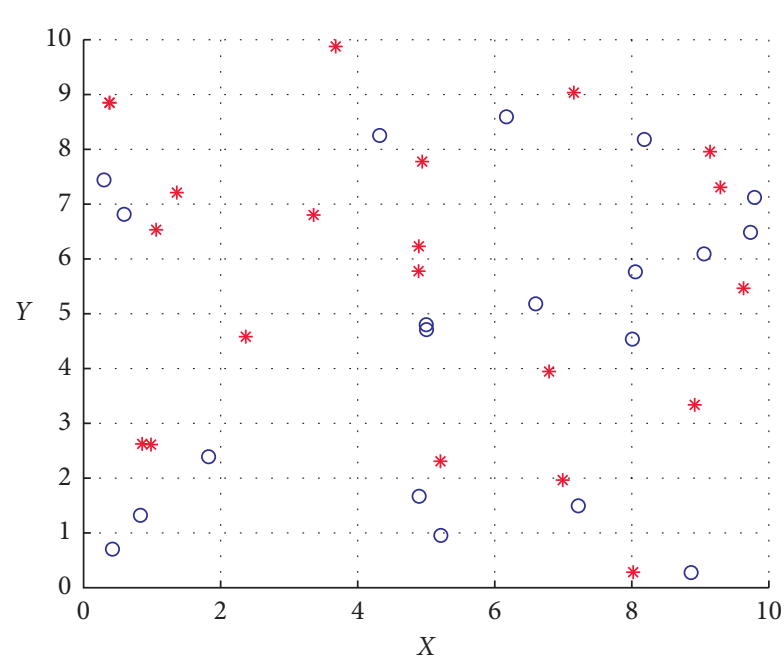

(a)

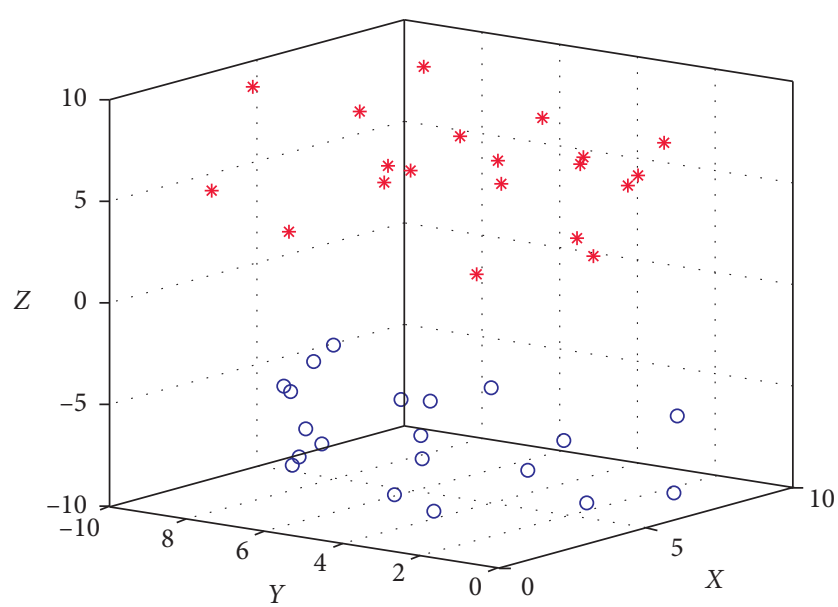

(b)

Figure 2: Nonlinear separable case. 


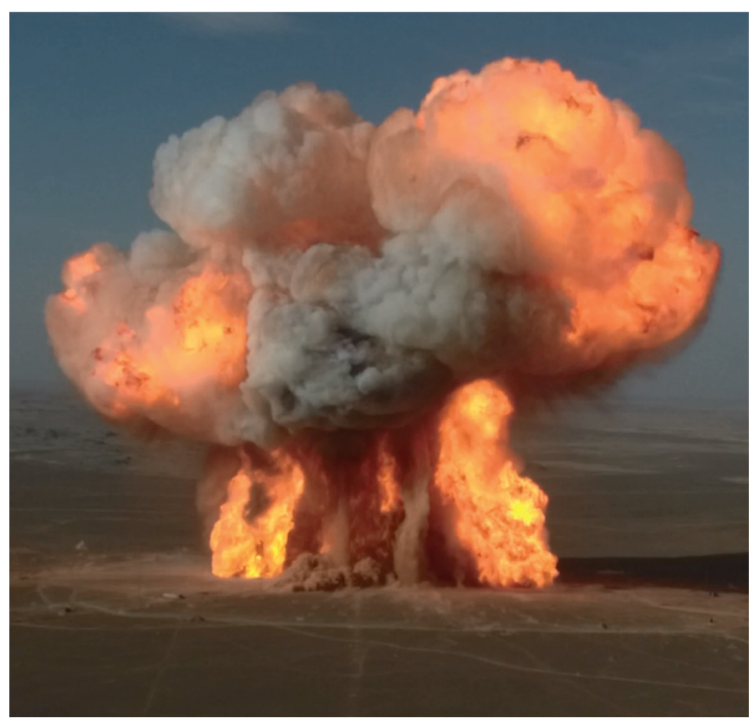

(a)

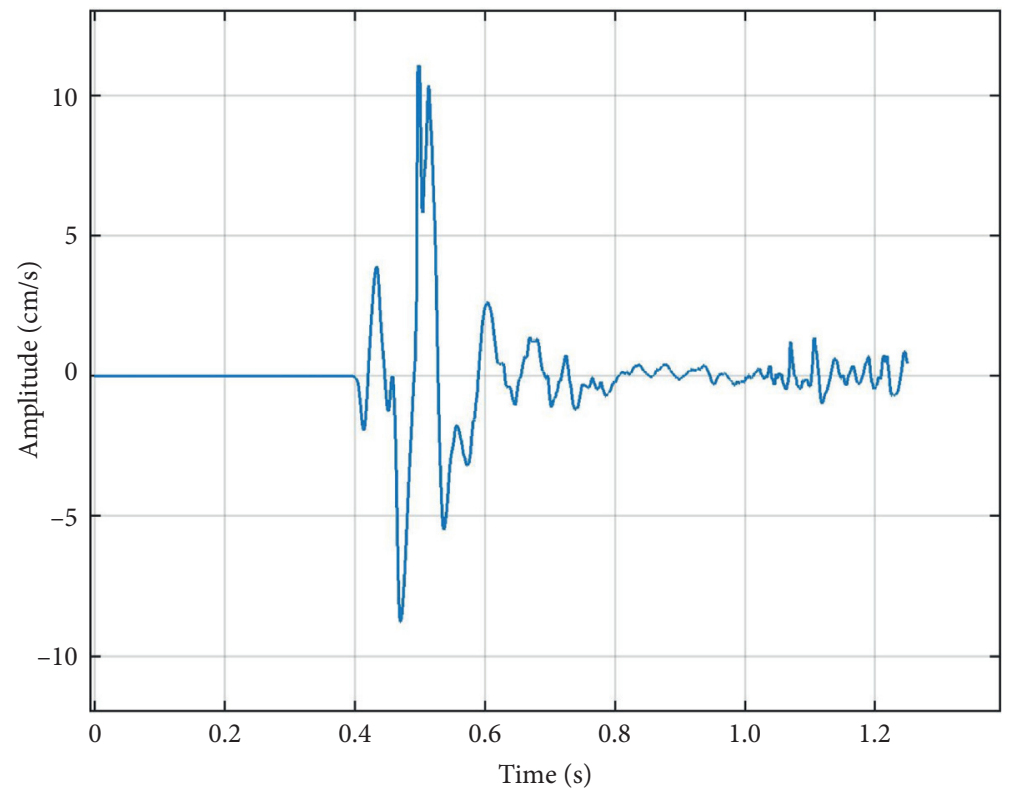

(b)

Figure 3: Pipeline explosion vibration. (a) OD1422-12Mpa explosion experiment of natural gas pipeline [26]. (b) Vibration signal.

large-scale infrastructure projects such as nuclear power plants, airports, tunnels, and ports. The high standard, high difficulty, and high strength of large-scale engineering construction put forward new challenges to the application of blasting technology. The impact of blasting vibration of subsequent projects on the projects that have been put into operation cannot be ignored. The research team obtained a large number of surface rock blast vibration data from the foundation pit excavation project of Fujian Fuqing Nuclear Power Plant for this study, as shown in Figure 5. The blast parameters are shown in Table 3.

Scene 4: blasting vibration in tunneling: because of its strong adaptability to geological conditions and low excavation cost, DBM (drilling and blasting method) is especially suitable for the construction of hard rock tunnel, broken rock tunnel, and a large number of short tunnels [27]. The vibration effect of DBM on the surrounding buildings, especially the subway lines which have been built and in operation, cannot be ignored. The research team had been stationed at the construction site of Nanjing Metro Tunnel for a long time, as shown in Figure 6, and collected the blasting vibration data of metro tunnel as a kind of sample for this study. The blast parameters are shown in Table 4.

The above data are collected by TC-4850 and Blast-UM vibration recorders, and the specific sampling parameters are shown in the table.

As mentioned above, the data samples in this study were divided into four categories, each of which takes 10 groups of data, 40 samples in total. In each training-prediction cycle, 


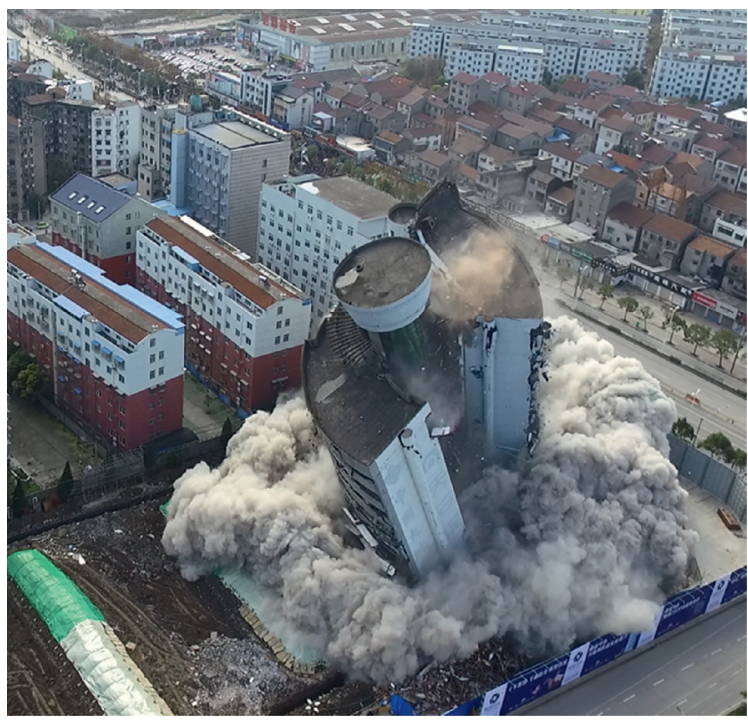

(a)

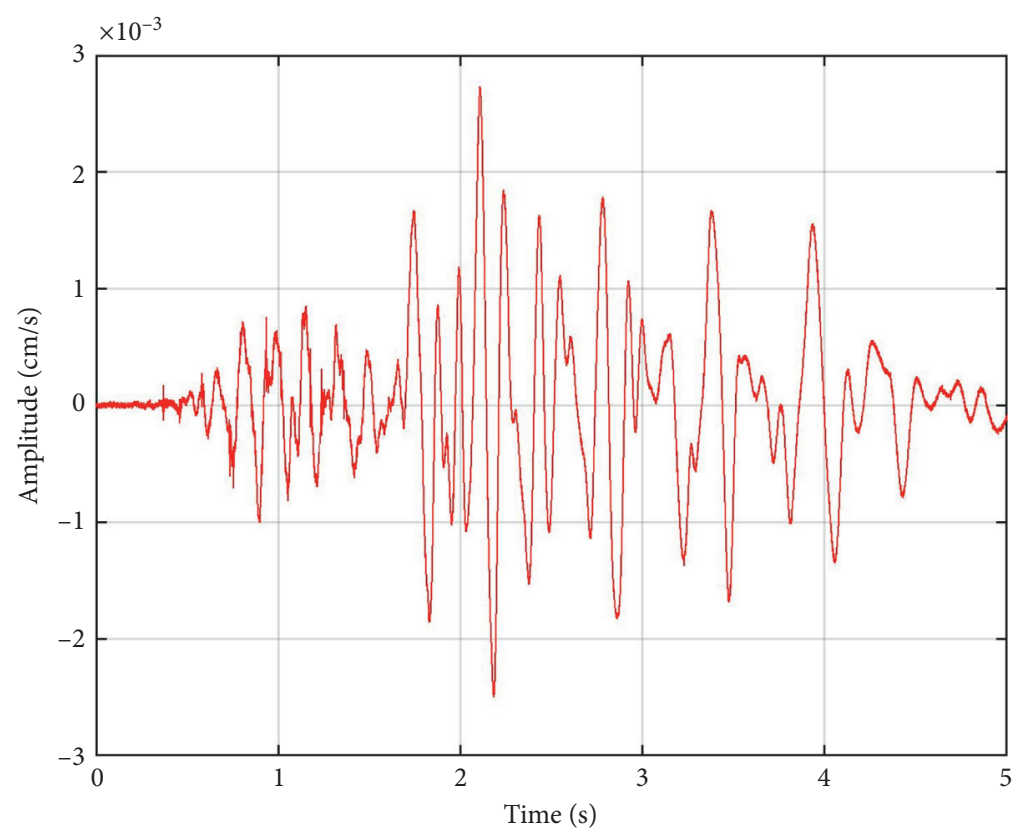

(b)

FIGURE 4: Demolition collapse vibration. (a) Blasting project of investment Plaza building in Jingzhou, Hubei Province. (b) Vibration signal.

30 groups of data were randomly selected as training sets, and the remaining 10 groups were detection sets. EEMD decomposition was carried out for each data. According to statistics, the number of IMF of 40 sample data was between 10 and 13. Considering that the main energy components were concentrated in the first several IMF layers, the CEE of the first nine IMF layers was taken and normalized to obtain the input matrix as shown in Table 5.

The feature matrix of $40 \times 9$ was input into OAO-SVM to train and test. The results are shown in Figure 7.

It can be seen from the figure that the prediction accuracy can be maintained above $80 \%$ in multiple training and tests. The prediction accuracy statistics of the four types of signals is shown in Table 6 .

It can be seen from the statistical results that this method has high prediction accuracy for underground tunnel blast vibration and surface rock blast vibration. On the other hand, this algorithm often misjudges the pipeline explosion vibration. The cause of this phenomenon may be that the sample size was too small.

The EEMD-SVM algorithm provides us with a new means for vibration classification. Compared with commonly used BP neural network algorithms, the new method has the characteristics of high accuracy and short training 


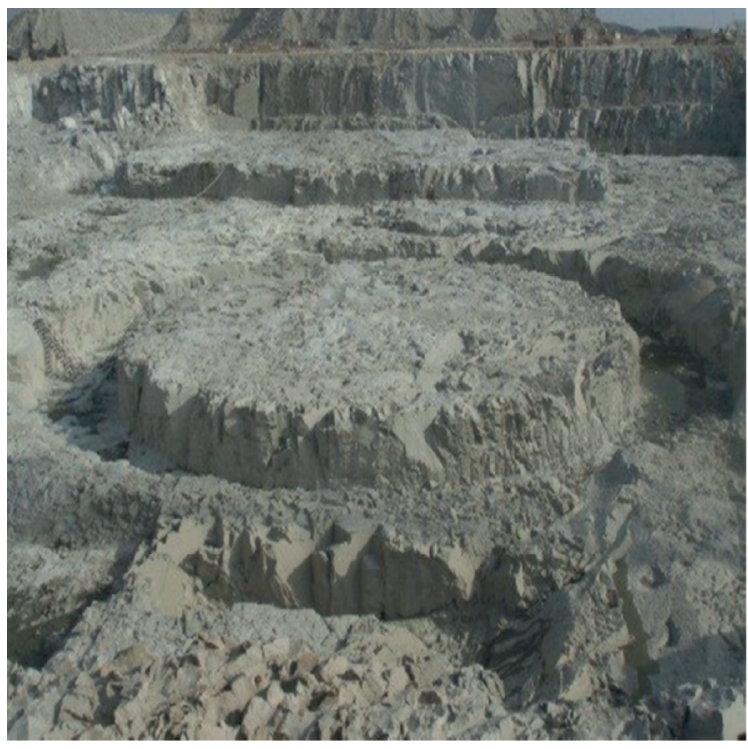

(a)

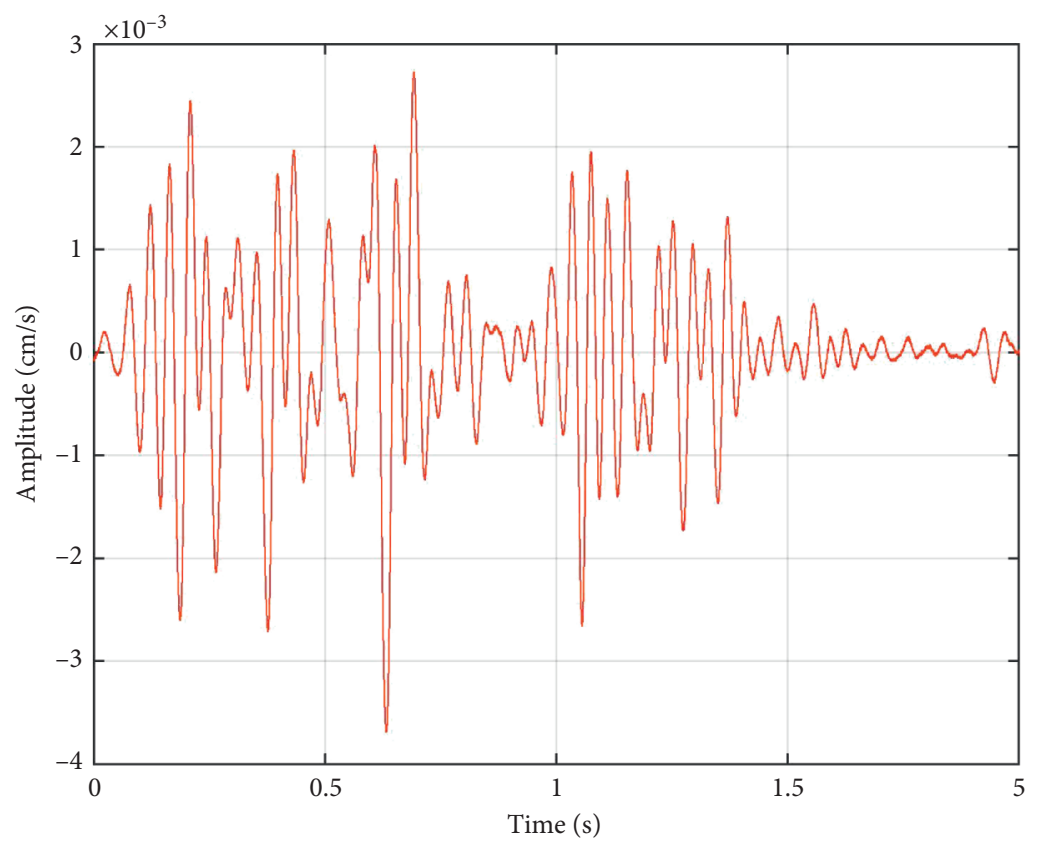

(b)

FIgURE 5: Surface rock blast vibration. (a) "Hualong No.1" foundation pit blasting project. (b) Vibration signal.

TABle 3: Parameters of rock blasting.

\begin{tabular}{lcccccc}
\hline $\begin{array}{l}\text { Hole spacing } \\
(\mathrm{m})\end{array}$ & $\begin{array}{c}\text { Row spacing } \\
(\mathrm{m})\end{array}$ & $\begin{array}{c}\text { Hole diameter } \\
(\mathrm{mm})\end{array}$ & $\begin{array}{c}\text { Hole depth } \\
(\mathrm{m})\end{array}$ & $\begin{array}{c}\text { Explosive mass } \\
(\mathrm{kg})\end{array}$ & $\begin{array}{c}\text { Resistance line } \\
(\mathrm{m})\end{array}$ & $\begin{array}{c}\text { Explosive } \\
\text { type }\end{array}$ \\
\hline 5.5 & 3.2 & 115 & 13 & 85 & 2.2 & $\begin{array}{c}\text { Delay } \\
\text { time } \\
(\mathrm{ms})\end{array}$ \\
\hline
\end{tabular}

time. The comparison between the two methods is shown in Table 7.

It can be seen from the table that SVM algorithm has certain advantages in prediction accuracy compared with neural network algorithm, while in training efficiency, it was much better than neural network algorithm.
Furthermore, the approximate entropy and permutation entropy are calculated on the basis of the inherent modal components as input eigenvectors, which are compared with the results of the algorithm in this paper, as shown in Table 8. It can be seen that, in terms of prediction accuracy and learning efficiency, the three algorithms are 


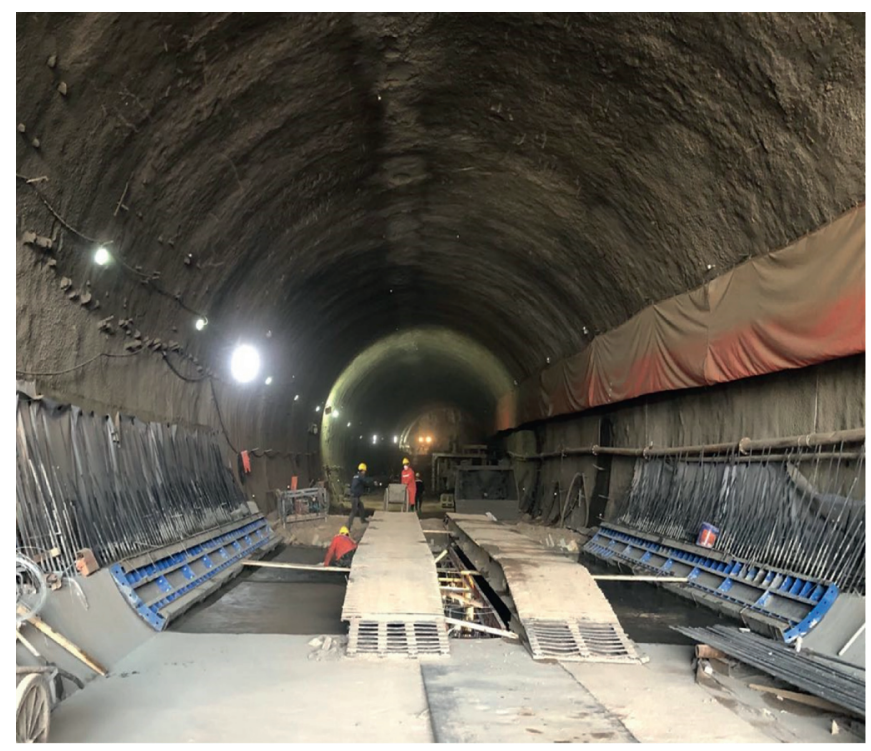

(a)

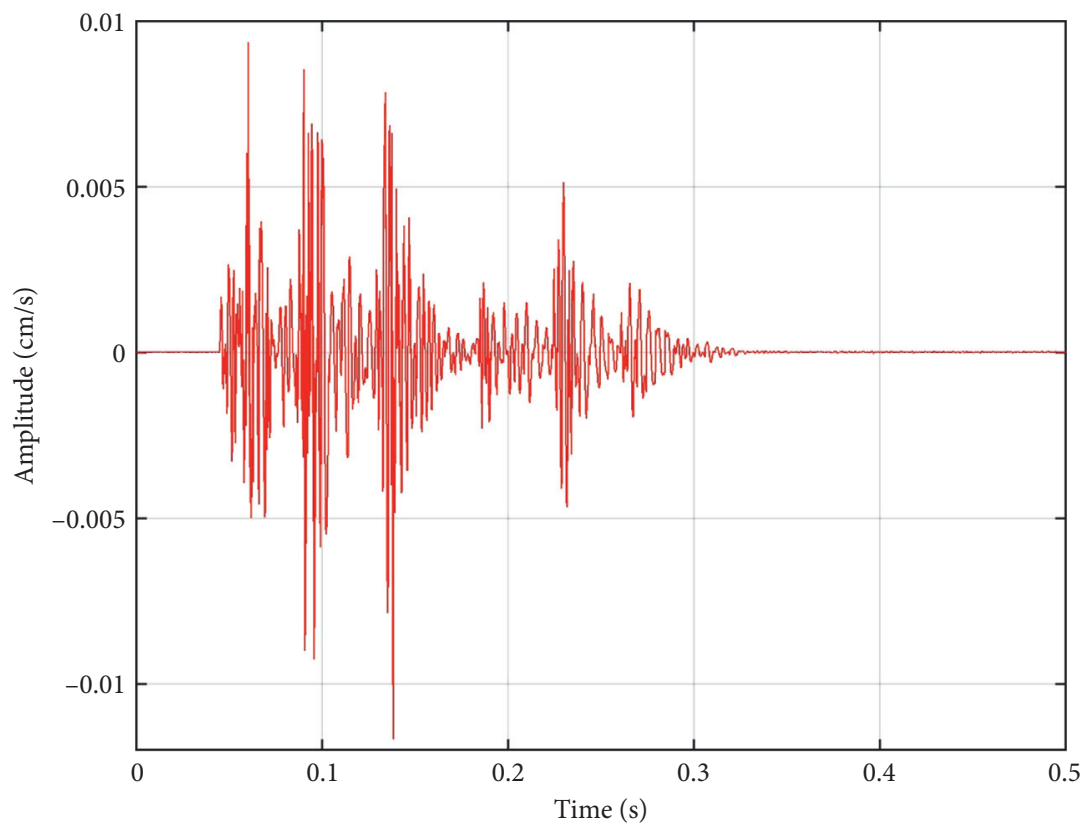

(b)

Figure 6: Underground tunnel blast vibration. (a) Blasting site of Nanjing metro line 3 tunnel. (b) Vibration signal.

TABle 4: Parameters of tunnel blasting.

\begin{tabular}{lcccccc}
\hline Blasting method Hole spacing $(\mathrm{cm})$ & Hole diameter $(\mathrm{mm})$ & Hole depth $(\mathrm{m})$ & $\begin{array}{c}\text { Specific consumption } \\
\left(\mathrm{kg} \cdot \mathrm{m}^{-3}\right)\end{array}$ & $\begin{array}{c}\text { Explosive type } \\
\text { time }(\mathrm{ms})\end{array}$ \\
\hline Cut blasting & 50 & 40 & 1.2 & $0.6 \sim 1$ & Emulsion explosive $\varphi 32$ & 50 \\
\hline
\end{tabular}

basically the same. However, due to the increase of approximate entropy and permutation entropy in the process of entropy calculation, the CPU time used in the construction of input eigenvectors is significantly increased, and it is highly related to the embedding dimension (permutation entropy) and reconstruction dimension (approximate entropy) parameters, so the comprehensive efficiency is at a disadvantage. 
TABLe 5: Input matrix.

\begin{tabular}{|c|c|c|c|c|c|c|c|c|c|c|}
\hline \multirow{2}{*}{ Signal } & \multirow{2}{*}{ Serial number } & \multicolumn{9}{|c|}{ Eigenvectors } \\
\hline & & $\mathrm{CEE}_{1}$ & $\mathrm{CEE}_{2}$ & $\mathrm{CEE}_{3}$ & $\mathrm{CEE}_{4}$ & $\mathrm{CEE}_{5}$ & $\mathrm{CEE}_{6}$ & $\mathrm{CEE}_{7}$ & $\mathrm{CEE}_{8}$ & $\mathrm{CEE}_{9}$ \\
\hline \multirow{10}{*}{ Pipeline explosion vibration } & 1 & 0.0279 & 0.0075 & 0.0819 & 0.2489 & 0.0217 & 0.2653 & 0.4851 & 0.5305 & 0.4968 \\
\hline & 2 & 0.0303 & 0.0163 & 0.0059 & 0.0062 & 0.0125 & 0.0290 & 0.0262 & 0.5166 & 0.4858 \\
\hline & 3 & 0.0828 & 0.0027 & 0.0420 & 0.0961 & 0.4683 & 0.3196 & 0.5111 & 0.4434 & 0.0963 \\
\hline & 4 & 0.0013 & 0.0282 & 0.2993 & 0.5303 & 0.4825 & 0.3942 & 0.3509 & 0.2898 & 0.0603 \\
\hline & 5 & 0.0011 & 0.0162 & 0.0989 & 0.4106 & 0.5162 & 0.5019 & 0.3201 & 0.1050 & 0.0597 \\
\hline & 6 & 0.0022 & 0.0221 & 0.0175 & 0.4069 & 0.5110 & 0.4941 & 0.3428 & 0.0698 & 0.1088 \\
\hline & 7 & 0.0006 & 0.0047 & 0.0493 & 0.0700 & 0.2878 & 0.3746 & 0.4724 & 0.5305 & 0.4554 \\
\hline & 8 & 0.0239 & 0.0036 & 0.0242 & 0.0733 & 0.4083 & 0.4934 & 0.4662 & 0.5040 & 0.3915 \\
\hline & 9 & 0.0236 & 0.0907 & 0.0412 & 0.0705 & 0.4006 & 0.5306 & 0.4053 & 0.4942 & 0.2492 \\
\hline & 10 & 0.0049 & 0.0172 & 0.1697 & 0.3350 & 0.3698 & 0.4949 & 0.4725 & 0.0911 & 0.0287 \\
\hline \multirow{10}{*}{ Underground tunnel blast vibration } & 11 & 0.5307 & 0.5300 & 0.3088 & 0.3942 & 0.0873 & 0.0439 & 0.0170 & 0.0097 & 0.0076 \\
\hline & 12 & 0.3478 & 0.3438 & 0.5261 & 0.5194 & 0.3942 & 0.1373 & 0.0281 & 0.0133 & 0.0115 \\
\hline & 13 & 0.3844 & 0.2399 & 0.4719 & 0.5265 & 0.5042 & 0.0853 & 0.0337 & 0.0118 & 0.0143 \\
\hline & 14 & 0.3990 & 0.4214 & 0.5307 & 0.4871 & 0.2307 & 0.1177 & 0.1023 & 0.0685 & 0.0239 \\
\hline & 15 & 0.2209 & 0.2740 & 0.5307 & 0.5300 & 0.3747 & 0.1071 & 0.0561 & 0.0226 & 0.0148 \\
\hline & 16 & 0.4189 & 0.4115 & 0.5188 & 0.5222 & 0.2383 & 0.0962 & 0.0981 & 0.0332 & 0.0099 \\
\hline & 17 & 0.5304 & 0.5026 & 0.2967 & 0.3365 & 0.3651 & 0.2369 & 0.1103 & 0.0315 & 0.0452 \\
\hline & 18 & 0.3332 & 0.3404 & 0.5173 & 0.4970 & 0.4597 & 0.2048 & 0.0867 & 0.0247 & 0.0322 \\
\hline & 19 & 0.3610 & 0.5030 & 0.4422 & 0.5287 & 0.2982 & 0.1074 & 0.0377 & 0.0463 & 0.0178 \\
\hline & 20 & 0.4228 & 0.5306 & 0.4153 & 0.3757 & 0.3952 & 0.1826 & 0.0860 & 0.0401 & 0.0257 \\
\hline \multirow{10}{*}{ Demolition collapse vibration } & 21 & 0.0031 & 0.0040 & 0.0016 & 0.0004 & 0.0016 & 0.1478 & 0.4680 & 0.4896 & 0.4417 \\
\hline & 22 & 0.0006 & 0.0001 & 0.0012 & 0.0024 & 0.1070 & 0.4057 & 0.2847 & 0.2110 & 0.0726 \\
\hline & 23 & 0.0016 & 0.0004 & 0.0001 & 0.0003 & 0.4343 & 0.3062 & 0.4134 & 0.1687 & 0.3587 \\
\hline & 24 & 0.0010 & 0.0003 & 0.0002 & 0.0001 & 0.0014 & 0.4967 & 0.2037 & 0.5247 & 0.3866 \\
\hline & 25 & 0.0001 & 0.0001 & 0.0000 & 0.0058 & 0.3794 & 0.4942 & 0.4950 & 0.3346 & 0.0972 \\
\hline & 26 & 0.0007 & 0.0003 & 0.0001 & 0.0001 & 0.0002 & 0.3554 & 0.4591 & 0.4919 & 0.4404 \\
\hline & 27 & 0.0006 & 0.0002 & 0.0001 & 0.0001 & 0.4003 & 0.3324 & 0.3792 & 0.2881 & 0.1832 \\
\hline & 28 & 0.0004 & 0.0003 & 0.0034 & 0.0204 & 0.0582 & 0.0786 & 0.4714 & 0.5305 & 0.2545 \\
\hline & 29 & 0.0006 & 0.0002 & 0.0001 & 0.0001 & 0.0069 & 0.5259 & 0.5142 & 0.2997 & 0.1634 \\
\hline & 30 & 0.0010 & 0.0002 & 0.0001 & 0.0003 & 0.1572 & 0.5283 & 0.5307 & 0.4387 & 0.3204 \\
\hline \multirow{10}{*}{ Surface rock blast vibration } & 31 & 0.0008 & 0.0003 & 0.0002 & 0.1390 & 0.4707 & 0.5269 & 0.2577 & 0.1172 & 0.0453 \\
\hline & 32 & 0.0010 & 0.0003 & 0.0002 & 0.5307 & 0.5108 & 0.5145 & 0.2442 & 0.0731 & 0.0359 \\
\hline & 33 & 0.0003 & 0.0001 & 0.0835 & 0.4339 & 0.5137 & 0.5259 & 0.1329 & 0.0407 & 0.0230 \\
\hline & 34 & 0.0003 & 0.0001 & 0.0125 & 0.1499 & 0.5250 & 0.5054 & 0.4730 & 0.1849 & 0.1713 \\
\hline & 35 & 0.0009 & 0.0004 & 0.0002 & 0.1870 & 0.5188 & 0.5298 & 0.3780 & 0.1559 & 0.0485 \\
\hline & 36 & 0.0006 & 0.0002 & 0.0085 & 0.1743 & 0.2947 & 0.3846 & 0.2294 & 0.0591 & 0.0183 \\
\hline & 37 & 0.0007 & 0.0005 & 0.0002 & 0.4827 & 0.5277 & 0.5270 & 0.1483 & 0.0348 & 0.0167 \\
\hline & 38 & 0.0026 & 0.0018 & 0.0008 & 0.0878 & 0.5184 & 0.5011 & 0.5090 & 0.0774 & 0.0280 \\
\hline & 39 & 0.0021 & 0.0011 & 0.0004 & 0.1693 & 0.4668 & 0.5307 & 0.1530 & 0.0472 & 0.0094 \\
\hline & 40 & 0.0054 & 0.0018 & 0.0011 & 0.0007 & 0.5202 & 0.5290 & 0.4917 & 0.2858 & 0.2168 \\
\hline
\end{tabular}

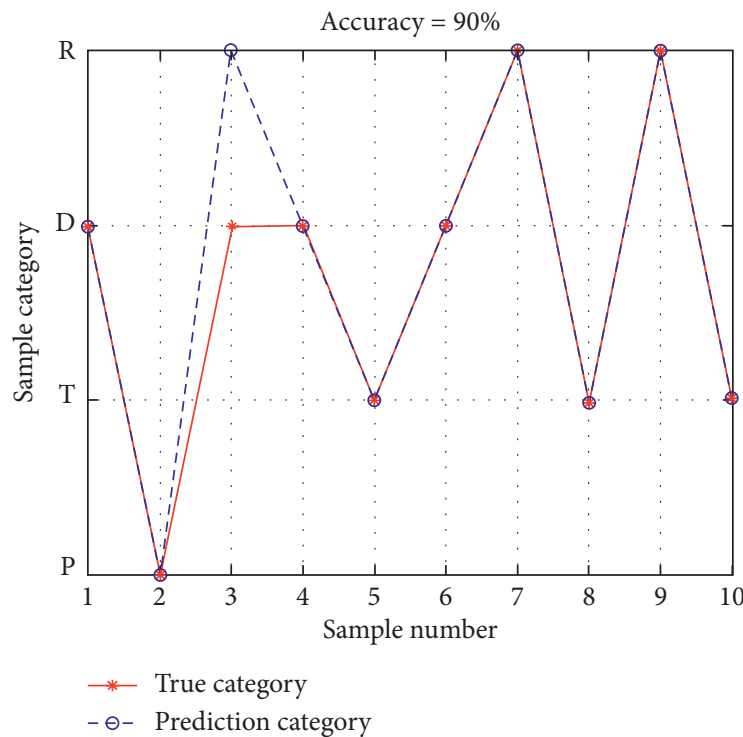

(a)

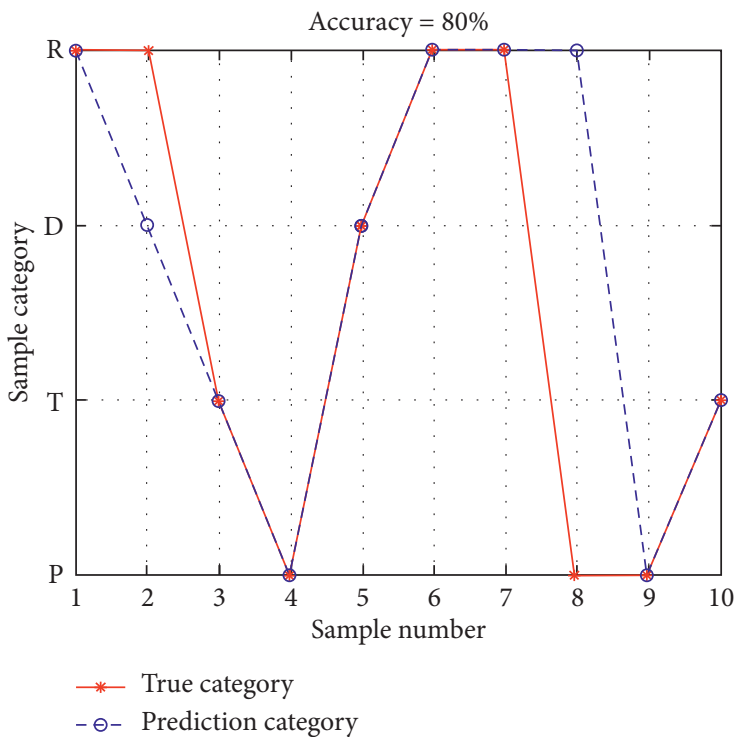

(b)

Figure 7: Continued. 


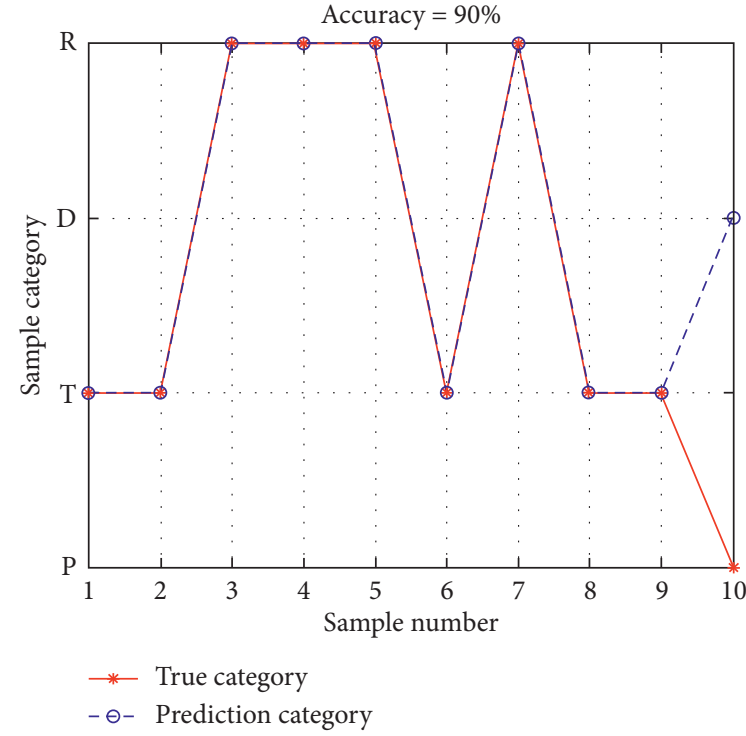

(c)

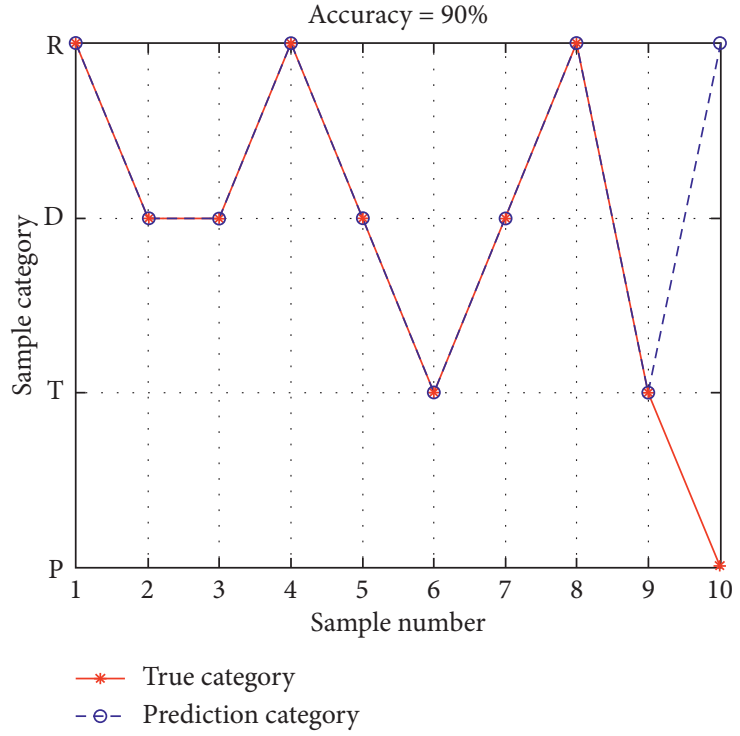

(d)

FIgURE 7: Comparison between the prediction results of SVM and the real situation. (a) First operation. (b) Second operation. (c) Third operation. (d) Fourth operation.

Table 6: Prediction result statistics.

\begin{tabular}{lcccc}
\hline Signal type & $\begin{array}{c}\text { Pipeline explosion } \\
\text { vibration (\%) }\end{array}$ & $\begin{array}{c}\text { Underground tunnel } \\
\text { blast vibration (\%) }\end{array}$ & $\begin{array}{c}\text { Demolition collapse } \\
\text { vibration }(\%)\end{array}$ & $\begin{array}{c}\text { Surface rock blast } \\
\text { vibration }(\%)\end{array}$ \\
\hline Prediction accuracy & 50 & 100 & 88.9 & 92.3 \\
\hline
\end{tabular}

TABLE 7: Algorithm effect comparison.

\begin{tabular}{lcccc}
\hline & \multicolumn{2}{c}{ SVM algorithm } & \multicolumn{2}{c}{ BP neural network algorithm } \\
& Prediction accuracy & Training time (s) & Prediction accuracy & Training time (s) \\
\hline Example 1 & 80 & 131 & 70 & 502 \\
Example 2 & 90 & 127 & 60 & 478 \\
Example 3 & 90 & 152 & 70 & 615 \\
\hline
\end{tabular}

TABLE 8: Algorithm effect comparison.

\begin{tabular}{lcccccc}
\hline & \multicolumn{2}{c}{ EEMD energy entropy } & \multicolumn{2}{c}{ Approximate entropy } & \multicolumn{2}{c}{ Permutation entropy } \\
& Prediction accuracy & Training time (s) & Prediction accuracy & Training time (s) & Prediction accuracy (\%) & Training time (s) \\
\hline Example 1 & 80 & 135 & 80 & 142 & 136 & 90 \\
Example 2 & 90 & 124 & 80 & 161 & 90 & 129 \\
Example 3 & 90 & 156 & 70 & 121 & 90 & 131 \\
Example 4 & 100 & 119 & 80 & 80 & 118 \\
\hline
\end{tabular}

\section{Discussion and Conclusion}

In this study, through the cross-research of EEMD algorithm and SVM algorithm, combined with the training and prediction of 4 types of typical explosion vibration signals, the relevant conclusions are obtained as follows:
(1) EEMD energy entropy can be used as a significant feature for explosion vibration signal recognition

(2) Compared with the traditional BP neural network algorithm, the method in this paper has greater advantages in training efficiency and prediction accuracy 
(3) Compared with permutation entropy and approximate entropy, the method in this paper has no obvious advantages in training efficiency and prediction accuracy, but the comprehensive efficiency of the algorithm has been improved.

The new algorithm can provide reference for urban vibration monitoring and help managers to know the type of accidents in a short time to make timely decisions.

Considering that the prediction accuracy of pipeline explosion vibration signal is insufficient, the author will further collect and sort out the data and expand the sample capacity, in order to achieve higher prediction accuracy.

\section{Data Availability}

The data used in this paper can be obtained in the following link: hyperlink https://figshare.com/s/185b350ad6803157536c.

\section{Conflicts of Interest}

The authors declare that they have no conflicts of interest regarding this work.

\section{Acknowledgments}

This research was supported by the National Natural Science Foundation of China (nos. 11672331 and 51608530). The authors would like to gratefully acknowledge this support.

\section{References}

[1] M. Wang, M. Zhong, L. Yuan et al., "Study on dynamic strain regularity and influencing factors of shallow buried metal pipe under collapse impact load," Shock and Vibration, vol. 2018, p. 11, Aricle ID 8792564, 2018.

[2] L. Lu, Y. Long, Q. M. Xie et al., "Decomposition and energy distribution of blasting vibration signal based on second generation wavelet packet," Explosion and Shock Waves, vol. 33, no. 2, pp. 140-146, 2013.

[3] D. W. Zhong, L. He, P. Cao et al., "Analysis of blasting vibration duration and optimizing of delayed time interval for millisecond blasting," Explosion and Shock Waves, vol. 36, no. 5, pp. 703-709, 2016.

[4] C. E. Shannon, "A mathematical theory of communication," The Bell System Technical Journal, vol. 27, pp. 379-423, 1948.

[5] A. Hunglinh, C. Junsheng, L. Kenli et al., "A roller bearing fault diagnosis method based on LCD energy entropy and ACROA-SVM," Shock and Vibration, vol. 2014, Article ID 825825, 12 pages, 2014.

[6] L. Y. Zhao, L. Wang, and R. Q. Yan, "Rolling bearing fault diagnosis based on wavelet packet decomposition and multiscale permutation entropy," Entropy, vol. 17, no. 9, pp. 6447-6461, 2015.

[7] Y. Li, X. Wang, Z. Liu, X. Liang, and S. Si, “The entropy algorithm and its variants in the fault diagnosis of rotating machinery: a review," IEEE Access, vol. 6, pp. 66723-66741, 2018.

[8] L. Li, Y. Y. Wang, and X. X. Li, “An improved wavelet energy entropy algorithm for speech endpoint detection," Computer Engineering, vol. 43, no. 5, pp. 268-274, 2017.

[9] F. Cheng-Wei, B. Guang-Chen, T. Wen-Zhong et al., "Quantitative diagnosis of rotor vibration fault using process power spectrum entropy and support vector machine method," Shock and Vibration, vol. 2014, Article ID 957531, 9 pages, 2014.

[10] Z. Zhou, R. Cheng, X. Cai, D. Ma, and C. Jiang, "Discrimination of rock fracture and blast events based on signal complexity and machine learning," Shock and Vibration, vol. 2018, Article ID 9753028, 10 pages, 2018.

[11] G. S. Hu, F. F. Zhu, and Z. Ren, "Power quality disturbance identification using wavelet packet energy entropy and weighted support vector machines," Expert Systems with Applications, vol. 35, no. 1-2, pp. 143-149, 2008.

[12] B. Pandey, "Testing isotropy in the Two Micron All-Sky redshift survey with information entropy," Monthly Notices of the Royal Astronomical Society, vol. 468, no. 2, pp. 1953-1961, 2017.

[13] C. Xu, C. Hu, X. Liu et al., "Information entropy in predicting location of observation points for long tunnel," Entropy, vol. 19, no. 7, 2017.

[14] V. Vapnik, Statistical Learning theory, vol. 3, pp. 401-492, Wiley, New York, NY, USA, 1998.

[15] Z. Zhou, Machine learning, pp. 121-139, Tsinghua University Press, Beijing, China, 2016.

[16] M. C. Ferris and T. S. Munson, "Interior-point methods for massive support vector machines," SIAM Journal on Optimization, vol. 13, no. 3, pp. 783-804, 2002.

[17] L. Bottou, "Large-scale machine learning with stochastic gradient descent," in Proceedings of COMPSTAT'2010, pp. 177-186, Springer, Berlin, Germany, 2010.

[18] H. Li, Statistical Learning methods, pp. 95-135, Tsinghua University Press, Beijing, China, 2012.

[19] O Stamm, Machine Learning Methods in the Environmental Sciences: Neural Networks and kernels, p. 193, Cambridge University Press, Cambridge, UK, 2009.

[20] C. W. Hsu and C. J. Lin, "A comparison of methods for multiclass support vector machines," IEEE Transactions on Neural Networks, vol. 13, no. 2, pp. 415-425, 2002.

[21] M. Gönen and E. Alpaydın, "Multiple kernel learning algorithms," Journal of Machine Learning Research, vol. 12, pp. 2211-2268, 2011.

[22] S. Fine and K. Scheinberg, "Efficient SVM training using lowrank kernel representations," Journal of Machine Learning Research, vol. 2, pp. 243-264, 2002.

[23] A. Ben-Hur and J. Weston, “A user's guide to support vector machines," in Data Mining Techniques for the Life Sciences, pp. 223-239, Humana Press, Totowa, NJ, USA, 2010.

[24] X. Yang, Q. Yu, L. He et al., "The one-against-all partition based binary tree support vector machine algorithms for multi-class classification," Neurocomputing, vol. 113, pp. 1-7, 2013.

[25] H. Ma, M. Zhong, X. Li, Q. Xie, Y. Zhou, and Q. Yin, "Experimental and numerical simulation study on the shock and vibration effect of OD1422-X80 mainline natural gas pipeline explosion," Shock and Vibration, vol. 2019, Article ID 6824819, 13 pages, 2019.

[26] M. Huayuan, L. Yuan, L. Xinghua et al., "Study on vibration characteristics of natural gas pipeline explosion based on improved MP-WVD algorithm," Shock and Vibration, vol. 2018, Article ID 8969675, 13 pages, 2018.

[27] J. Zhang, X. Cao, S. Zheng et al., "Experimental study on ground vibration effect of shallow tunnel excavation blasting," Journal of Rock Mechanics and Engineering, vol. 24, no. 22, p. $4158,2004$. 